\title{
Engineering of Ribosome-inactivating Proteins for Improving the anti-HIV Efficacy
}

Jia-qi Lu ${ }^{1}$, Yong-Tang Zheng ${ }^{2}$ and Pang-Chui Shaw ${ }^{1^{*}}$

${ }^{1}$ Centre for Protein Science and Crystallography, School of Life Sciences,

The Chinese University of Hong Kong, Shatin, N.T., Hong Kong, China

${ }^{2}$ Kunming Institute of Zoology, Chinese Academy of Sciences, Kunming, China

*E-mail address: pcshaw@cuhk.edu.hk

\section{Introduction}

Ribosome-inactivating proteins (RIPs) are N-glycosidases. They depurinate A-4324 in rat 28S ribosomal RNA in the conserved $\alpha$-sarcin/ricin loop ( $\alpha$-SRL) and cease protein synthesis. Our group has shown that the internal peptide of the maize RIP precursor reduced the anti-HIV activity of the protein in infected macaque peripheral blood mononuclear cells (PBMC) and SHIV 89.6-infected Chinese rhesus macaque. We made use of the switch-on mechanism of maize RIP to incorporate HIV-1 protease recognition sequences to its internal inactivation region. Upon activation of this engineered maize RIP by HIV-1 protease in HIV-infected cells, the N-glycosidase activity and inhibitory effect on p24 antigen production in vitro and in infected human T cells were enhanced. This switch-on mechanism can also be applied to ricin A chain (RTA). RTA variants with HIV-1 protease recognition sequence at the C-terminus can be cleaved both in vitro and in HIV-infected cells. Furthermore, its antiviral effect was enhanced and the cytotoxicity towards uninfected cells was reduced. Our study provides a platform technology in creating protein toxin derivatives with increased pathogen-specific cytotoxicity.

\section{Results}

Table 1. Cytotoxicity and antiviral activities of maize ribosome inactivating protein (RIP) variants tested on rhesus macaque peripheral blood mononuclear cells (PBMC).

\begin{tabular}{ccccc}
\hline \multirow{2}{*}{ RIP variants } & Cytotoxicity $\mathbf{C C}_{50}(\boldsymbol{\mu M})$ & & p27 Antigen reduction EC $_{\mathbf{5 0}}(\boldsymbol{\mu M})$ \\
\cline { 2 - 3 } \cline { 5 - 5 } & Uninfected & & SHIV89.6 & SIVmac239 \\
\hline His-TAT-Pro & $>15$ & & $>24.85$ & $>24.85$ \\
His-TAT-MOD & $8.98 \pm 0.36$ & & 5.53 & 11.23 \\
\hline
\end{tabular}
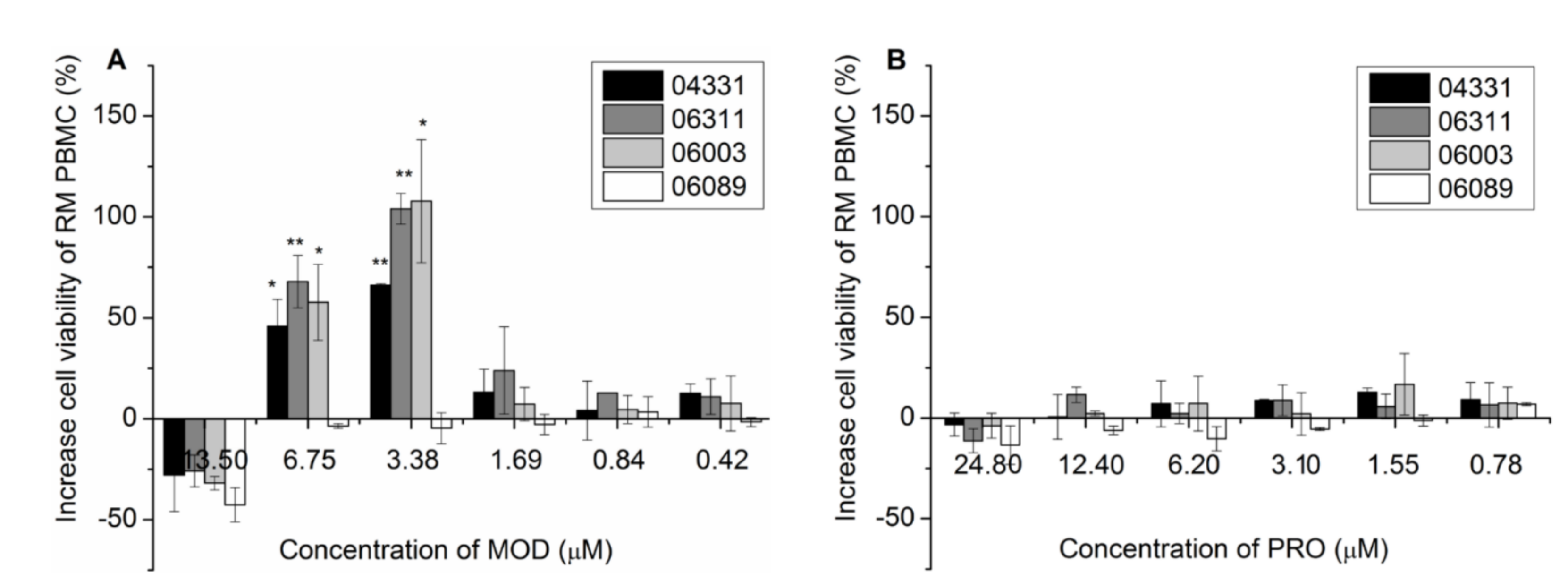

C
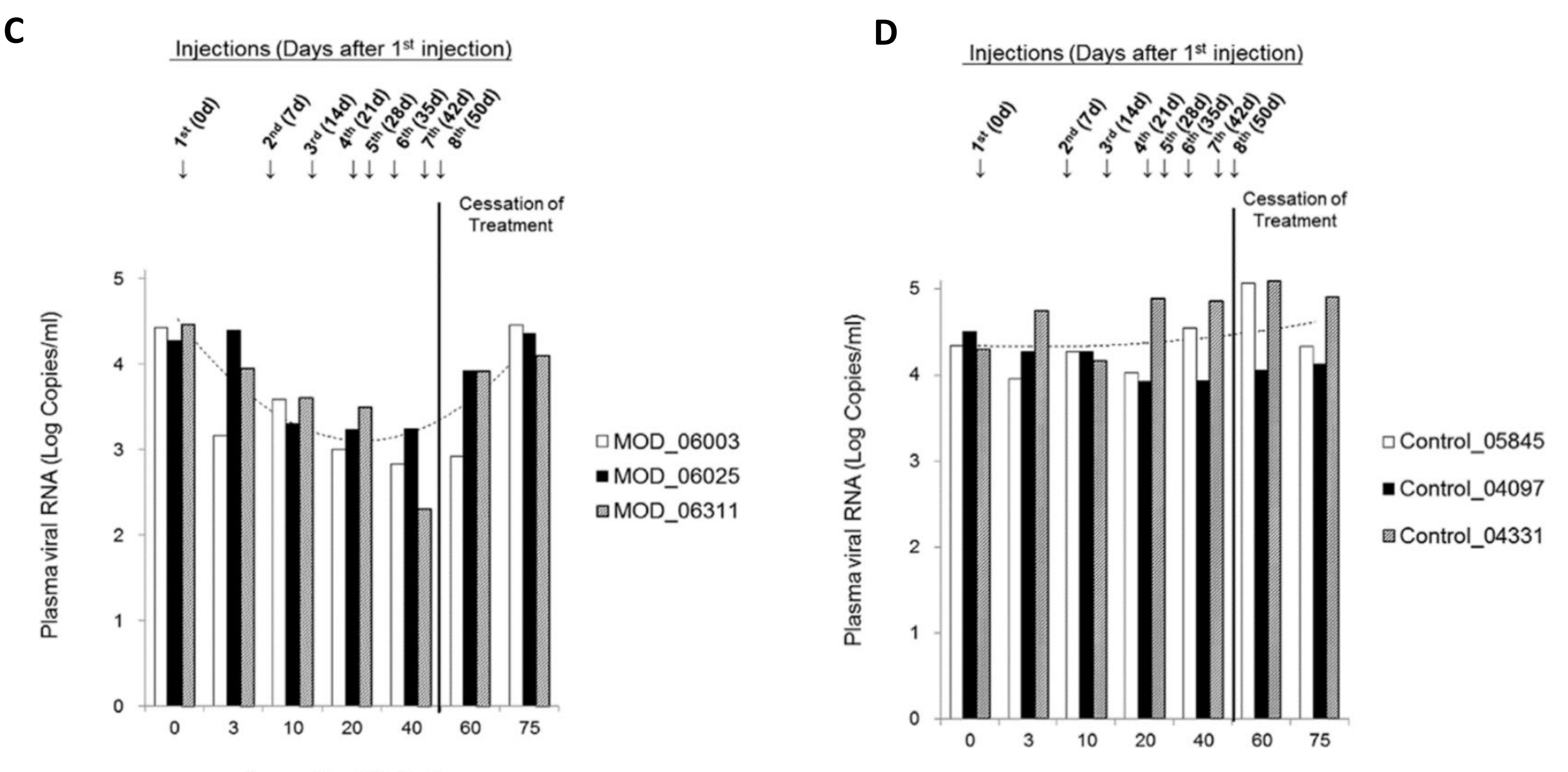

Figure 1 Antiviral Activities of Maize RIP in Rhesus Macaques

Protection for simian-human immunodeficiency virus (SHIV)-induced lysis on SHIV89.6-infected macaque PBMC upon treatment of (A) His-TAT-MOD; and (B) His-TAT-Pro. The experiment was repeated three times and mean $\pm S D$ was calculated for graphic presentation. Paired T-test was used for statistical analysis $\left({ }^{*} p<0.05,{ }^{* *} p<0.01\right)$. Plasma SIV viral load in rhesus macaques treated with (C) His-TAT-MOD; and (D) normal saline as negative control. Each group consisted of three macaques and "---" represented trend-line plotted using the average value from three individuals.

A Pro-RIP $(30.4 \mathrm{kDa})$ $\operatorname{MOD}(27.9 \mathrm{kDa})$

TAT-Pro-HIV-p2/NC (31.5 kDa) TAT-Pro-HIV-MA/CA (31.6 kDa)

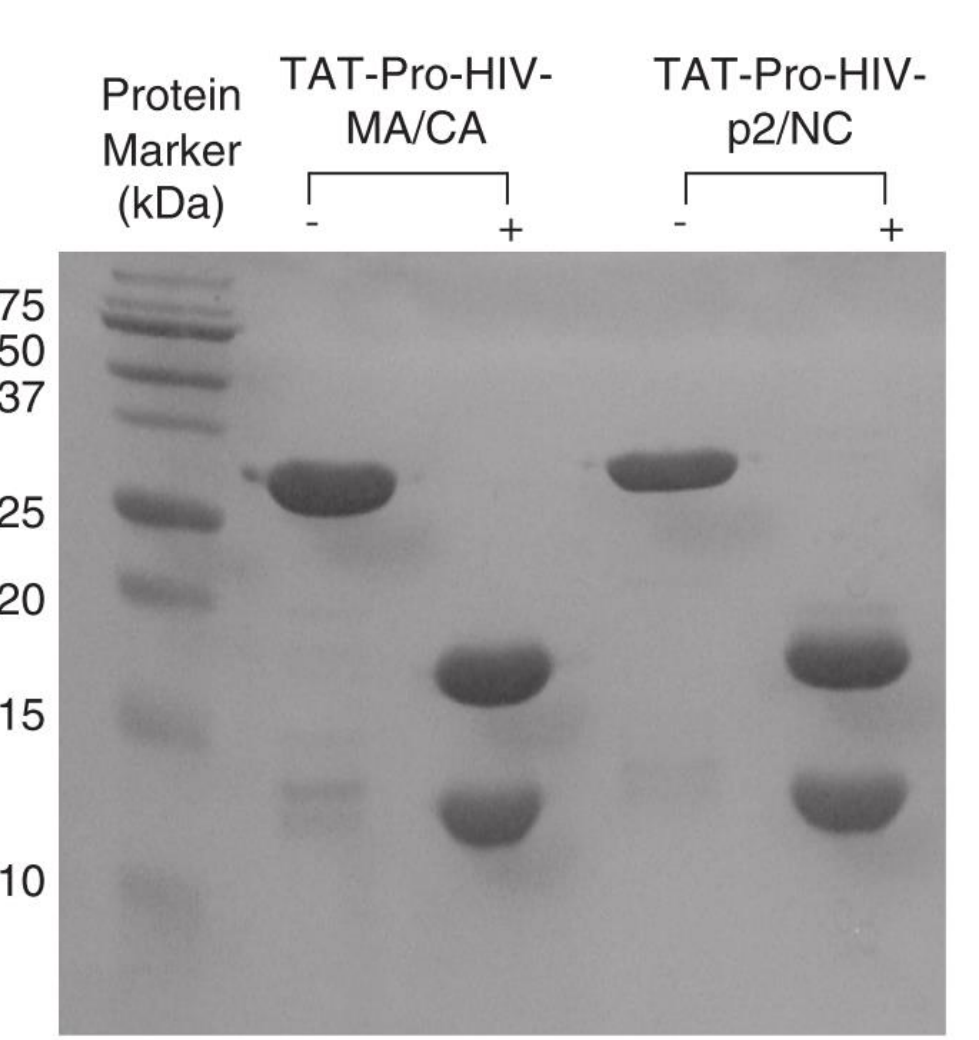

\section{Figure 2 A switch-on mechanism to activate maize ribosome-} inactivating protein for targeting HIV-infected cells

(A) Schematic diagram of the maize RIP and recombinant variants. (B) Cleavage of TAT-fused maize RIP variants by HIV-1 protease. TAT-Pro-HIV-MA/CA and TAT-Pro-HIV-p2/NC were completely cleaved by HIV-1 protease in vitro. (C) HIV-1IIIB acutely infected C8166 cells $\left(1 \times 10^{6}\right)$ were incubated with protein samples $(0.4 \mathrm{mg}$ in a volume of $2 \mathrm{ml})$ for $72 \mathrm{~h}$ and immunoblotted with anti-MOD polyclonal antibodies specific for Pro-RIP and its cleavage fragments $(\sim 11$ and $17 \mathrm{kDa})$.

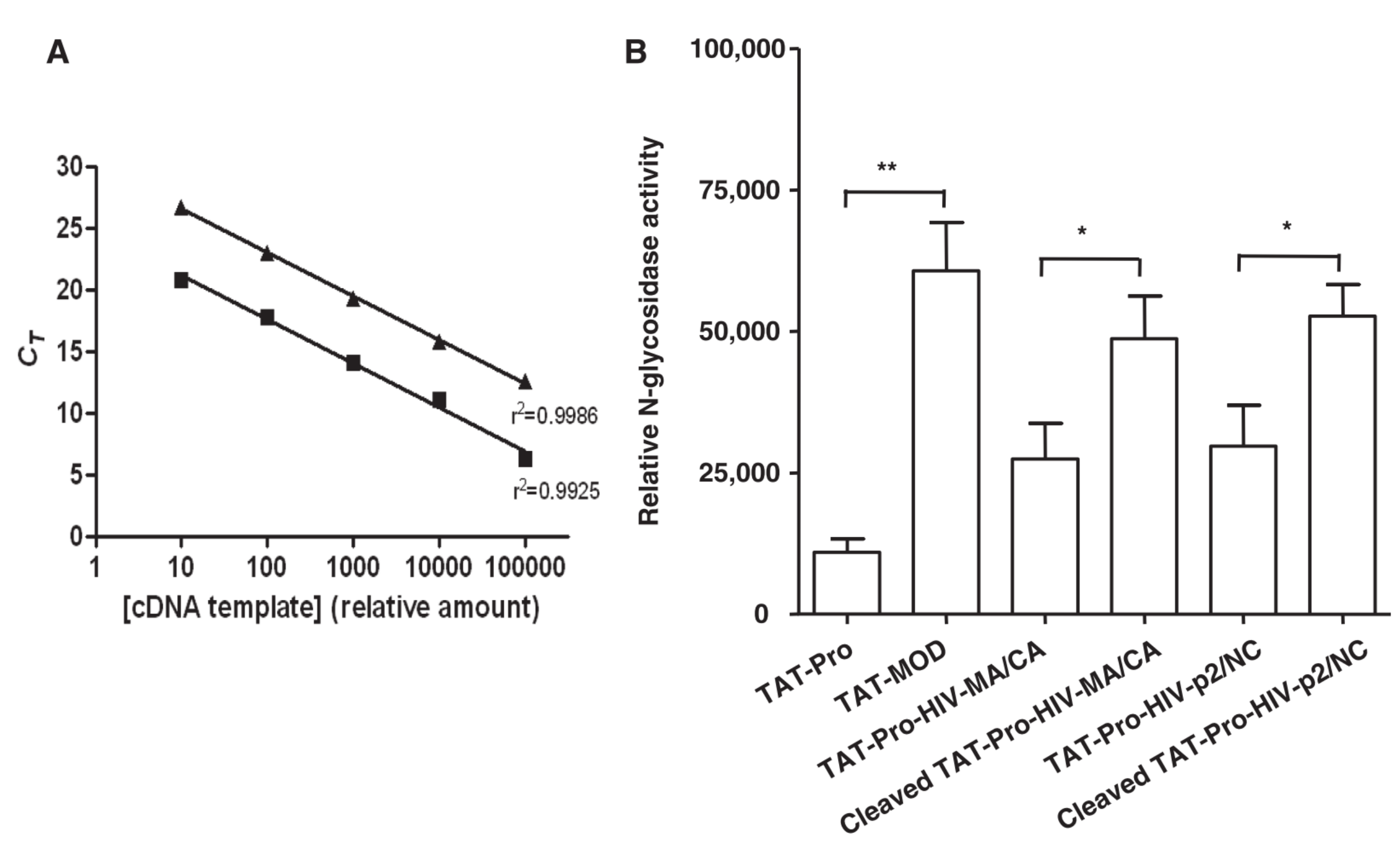

Figure 3 In vivo N-glycosidase activity of TAT-fused maize RIP and variants in mouse macrophage 28S rRNA (J774A.1)

During first-strand cDNA synthesis, reverse transcriptase preferentially inserts an adenine at the site of depurination, resulting in a $T$ to $A$ transversion in sequencing reads. $\mathrm{N}$-glycosidase activity was determined by qPCR using primers that target the modified site. (A) qRT-PCR efficiency test for primer pairs. (B) Relative N-glycosidase activity of the TAT-fused maize RIP and variants. The relative $\mathrm{N}$-glycosidase activity was calculated as the relative amount of altered rRNA of sampletreated cells over the untreated cells and mean \pm SEM was calculated for the graphic presentation. Unpaired t-test was performed for statistical analysis $(n=6)\left({ }^{\star} P<0.05,{ }^{* *} P<0.01\right)$.

\section{A}

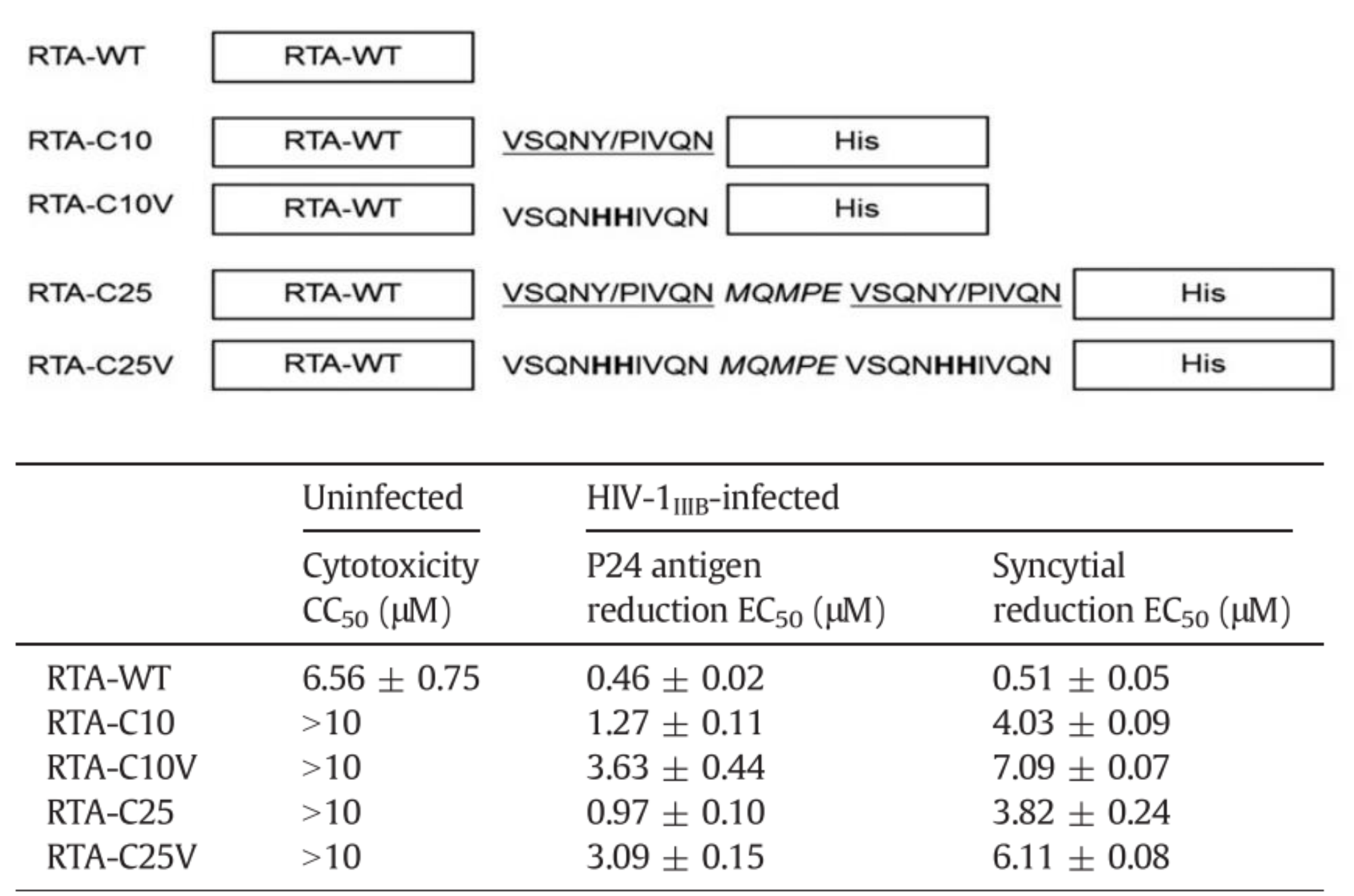

Figure 4 Engineering a switch-on peptide to ricin A chain for increasing its specificity towards HIV-infected cells

(A) Design and preparation of RTA variants. RTA-C10 and RTA-C25 were generated by inserting one and two HIV-1 specific sequences respectively at C-terminus of RTA in prior to His-tag. RTAC10V and RTA-C25V are the corresponding non cleavable counterparts with two middle residues of sequence modified to $\mathrm{HH}$ (bolded). (B) Cytotoxicity and antiviral activity of C-terminal variants in uninfectedandHIV-1IIIB acutely infected C8166 cells. The values are presented as means \pm SD ( $n$ $=6)$.

\section{Conclusion}

In this study, we found that the recombinant active maize RIP protected chimeric simian-human immunodeficiency virus (SHIV) 89.6-infected macaque peripheral blood mononuclear cells from lysis ex vivo and transiently reduced plasma viral load in SHIV89.6-infected rhesus macaque model. Besides, a switch-on strategy was applied on maize RIP and RTA based on the incorporation of HIV-1 protease recognition sequences to their internal inactivation region for activating the enzymatic activity of maize RIP in target cells, which provides a platform for combating pathogens with a specific protease.

\section{References}

Wang, R.R., et al. The recombinant maize ribosome-inactivating protein transiently reduces viral load in SHIV89.6 infected Chinese Rhesus Macaques. Toxins (Basel) 2015, 7, 156-169 